http://jmscr.igmpublication.org/home/ ISSN (e)-2347-176x ISSN (p) 2455-0450

crossref DOI: https://dx.doi.org/10.18535/jmscr/v8i8.21

\title{
Types of Liver Abscesses - A Prospective Observational Study in Sub-Himalayan Region
}

\author{
Authors \\ Purnima Patial ${ }^{1}$, Karan S. Thakur ${ }^{1}$ \\ Corresponding Author \\ Karan S. Thakur
}

\begin{abstract}
Liver is a significant and fundamental organ of the body. This organ is exposed to various infections; viral, bacterial and parasitic and lies at the distal end of portal flow; it is therefore washed with entrance blood containing infections, microorganisms, parasites, ova, results of absorption and other antigens.48\% of all visceral abscesses is liver abscess. The two most normal reasons for liver abscesses is the amoebic and pyogenic liver abscess. In our case report of 73 patients likewise, the two most basic causes also came out to be ALA and PLA. Liver abscess is still a diagnostic challenge in today's era. This is reflected in noteworthy death rates and is an aftereffect of the absence of particularity of clinical signs and research center outcomes. Amoebic and pyogenic abscess share numerous clinical, lab and imaging features, but they differ in their epidemiology and diseases transmission also. Thus differentiation is required for effective management and treatment.

Keywords: ALA - Amoebic liver abscess, PLA - Pyogenic liver abscess, E. histolytica Entamoebahistolytica, TB-Tuberculosis.
\end{abstract}

\section{Introduction}

Liver is a significant organ of the body. In the present era of quickly developing modern medicine, the liver is as yet the principle focal point of attention for some abdominal problems. In the abdominal infections and intra abdominal abscesses, the fundamental attention of the physician additionally ought to be the liver as the liver is the organ generally subjected to the development of abscess as it is $48 \%$ of all the visceral abscesses. Liver abscesses make up $13 \%$ of the complete number of abscesses. India has second highest incidence of liver abscess in the world.
The normal etiological agents for liver abscess are E. histolytica (amoebic), bacterial (pyogenic), Mycobacterium tuberculosis, and different parasites. Out of all incidences of liver abscess, approximately $66 \%$ of cases in developing countries are of amoebic etiology and $33 \%$ of cases in developed countries are pyogenic.

Amoebiasis is presently the third most common cause of death from parasitic disease. Amoebic liver abscess represents 3-9\% of all cases of amoebiasis. Amoebiasis is an infection caused by the intestinal protozoan Entamoeba histolytica. Amoebic liver abscess (ALA) is the commonest extra-intestinal manifestation of amoebic infection. 
PLA is generally encountered in clinical practice and carries huge mortality. In terms of causative pathogens, bacteria most frequently associated with PLA are Escherichia coli, Enterobacteriaceae family, anaerobes, and other members of the gastrointestinal flora. Over the previous 2 decades Klebsiella pneumoniae has been emerging as the predominant pathogen responsible for half to $88 \%$ of PLA in the Asian population.

The incidence of tubercular liver abscess has likewise increased in recent past. Typically the main focus remains elsewhere like lung or gastrointestinal tract and tubercle bacilli gets disseminated hematogenously and gets lodged into liver parenchyma.

\section{Case Study}

It is a prospective observational study, conducted in Department of Internal Medicine, Dr. R.P.G.M.C. Tanda. In this all patients diagnosed with liver abscess who were admitted in Medicine ward from $1^{\text {st }}$ January 2018 to $31^{\text {st }}$ December 2018 were taken as the sample size. Total of 73 patients of liver abscess got admitted.

\section{Case Definitions}

Amoebic liver abscess defined as a hepatic lesion finding on USG and/or CT- scan in a patient with significant clinical findings (various symptoms like fever, pain right hypochondrium, and abnormal liver function tests), plus one or more of the following:

(a) Recovery of amoebas from the aspirate, or

(b) A positive amoebic serology of more than 1:256 by indirect hemagglutination test according to laboratory values, or

(c) Clinical response to antiamoebic treatment.

A pyogenic liver abscess defined as a hepatic lesion demonstrated at Ultrasound (US) and/or computed tomography (CT) in a patient with a significant clinical findings (various symptoms suggestiveof sepsis, pain right hypochondrium, and abnormal liver function tests), plus one or more of the following: (a) A positive culture result from aspiration of the lesion, or

(b) A positive blood culture result, or

(c) Clinical response to antibiotics.

Aspirate was collected in sterile container and was sent to microbiology department for microscopic examination of:

- Wet Mount for trophozoites of Entamoeba histolytica

- Gram Staining

- $\mathrm{ZN}$ staining for AFB

- Culture sensitivity

\section{Observations and Results}

Between January 1, 2018, and December 30, 2018, 73 patients with liver abscess who were admitted in department of medicine were screened.

Out of 73 patients, 58 (79\%) patients suffered from ALA and 15 (20\%) patients suffered from pyogenic liver abscess and in this study no patient had fungal or tubercular abscess.

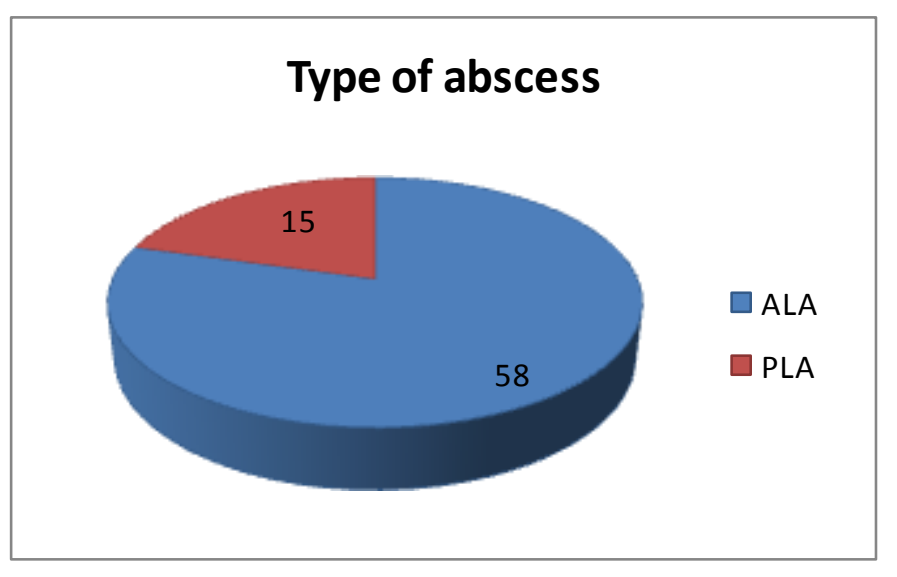

\section{Details of Causative Organis ms}

Fifty eight patients (79\%) had positive amoebic serology with titre of more than 1:256 by indirect hemagglutination test. No amoebas were recovered from any pus aspirate.

Out of 15 (21\%)patients of PLA, 7 patients had positive pus culture for E.coli,2 patient had positive pus culture for MRSA and 1 patient was positive for MSSA on pus culture. 2 patients had positive blood culture for MRSA.3 patients did 
not have either of blood culture or pus culture positive for any microorganism.

\begin{tabular}{|l|c|c|}
\hline & ORGANISM & No of patients \\
\hline Pus culture & E.coli & $7(47 \%)$ \\
& MRSA & $2(13 \%)$ \\
& MSSA & $1(7 \%)$ \\
\hline Blood culture & MRSA & $2(13 \%)$ \\
\hline & Sterile & $3(20 \%)$ \\
\hline TOTAL & & $15(100 \%)$ \\
\hline
\end{tabular}

\section{Discussion}

Liver abscess has been described since Hippocrates (400BC) and Ochsner (1936) and mortality was very high during those days i.e. upto $70-90 \%$.But the mortality rate has come down to 5-30\% with new diagnostic and microbiological techniques and newer management drainage procedures.

In our study 58 (79\%) patients were found to have ALA. Amoebic serology came out to be positive (> 1:256 titres) in 58 out of 73 patients of liver abscess in our study. The serum antibodies to $E$. histolytica become positive after one week of symptoms onset and may remain positive for more than 6 months. Positive results in association with the significant clinical symptoms suggest active disease. There are high chances of seropositivity for amoebiasis (owing to previous infection with E. histoloytica) which may complicate the interpretation of a positive serological test.

In our study,15 (21\%) patients were diagnosed with PLA.Among PLA patients, the most common organism identified in our study was E.coli in $47 \%$ patients. 3 patients did not have either of pus culture or blood culture positive. This may be due to partially treated infection with antibiotics before admission to our hospital.

Liver abscess can present with multiple clinical presentation. Both amoebic liver abscess and pyogenic liver abscess have almost similar clinical features. Amoebic liver abscess is more common than pyogenic liver abscess in our community due to its endemicity. Timely diagnosis of liver abscess is required to prevent complications and mortality.

\section{References}

1. Sharma N, Sharma A, Varma S, Lal A, Singh V. Amoebic liver abscess in the medical emergency of a North Indian hospital; BMC Research Notes.2010; vol. 3, article 21.

2. Das A, Saikia A, Saikia A.M., Dutta N. Clinico-epidemiological Profile of Patients with Liver Abscess: A Hospital Based Study; Indian Journal of Basic and Applied Medical Research; December 2015,5(1), P. 17-25.

3. Huston C, "Intestinal protozoa," in Feldman: Sleisenger Fordtran's Gastrointestinal and Liver DiseasePathophysiology, Diagnosis and Management, M. Feldman, L. S. Friedman, L. J. Brandt, and M. H. Sleisenger, Eds., vol. 8, pp. 2414-2419, Saunders Elseviers, Philadelphia, $\mathrm{Pa}$, USA, 2006

4. Barshak M, Kasper D. Intraabdominal Infections and Abscesses; Harrison's Principles of Internal Medicine by Kasper et al.19th edition, volume 2,page-850.

5. Abbas T, Khan Y, Muhsin A, Al-Dehwe B, Abukamar M, Elzouki A. Epidemiology, Clinical Features and Outcome of Liver Abscess: A single Reference Centre Experience in Qatar; Oman Medical Journal. 2014;29(4):260263. 